Lehner and colleagues reply -- We appreciate the alternative interpretation of our data suggested by Gordon Ada and Arno Müllbacher. Complete or partial protection from rectal mucosal SIV infection was found in all seven immunized macaques by targeting the vaccine in the proximity of the iliac lymph nodes that drain the recto-genital mucosa, but not by delivering the same vaccine by two other routes'. Administration of the vaccine near the lymph node must therefore be significant in eliciting protective immunity, and one of the determining factors might well be the antigenpresenting cells in and near the lymph node. However, one of the eight unimmunized control macaques failed to be infected rectally. A feature common to the protected immunized and unimmunized macaques was a significant increase in CD8-suppressor factor and the associated $\beta$-chemokines RANTES, MIP- $1 \alpha$ and MIP-1 $\beta$. These findings are open to at least four interpretations: (1) cross-reactive antigenic immune response $\mathrm{A}^{4}$; (2) specific $\mathrm{CD} 8^{+}$memory cells for which there can be little support in the naive control macaque; (3) nonspecific (unrelated) antigenic activation of $\mathrm{CD}^{+}$memory cells can be elicited by the CD28-CD80 noncognate second signal to stimulate the immune response ${ }^{2}$; and (4) proliferation of bystander $\mathrm{CD}^{+}$cells stimulated by interferons $\alpha$ and $\beta$, released by nonspecific antigenic activation $^{3}$. We are clearly unable to differentiate between these interpretations, and some of them may overlap. However, the experiment Ada and Müllbacher suggest is important. Indeed, we have arrived at a similar experiment via different reasoning. Namely, any nonspecific antigen administered by the TILN route might be capable of stimulating the three $\beta$-chemokines and inducing protection, if these chemokines are the only protective molecules within CD8$\mathrm{SF}$. There is, as yet, no confidence that CD8-SF (or CAF) can be accounted for entirely by those $\beta$-chemokines ${ }^{5}$. There are at least two other candidates: IL-16 (ref. 6) and stromal cell-derived factor $(\mathrm{SDF})^{7,8}$, quite apart from modulation of the corresponding CCKR5 and CXCKR4 receptors. It would, however, not be prudent to discard the SIV-specific secretory and serum antibodies and T-cell helper and cytotoxic responses that are involved in protection at three defined barriers: the mucosa, lymph node and circulation.
T. LeHNER ${ }^{1}$, Y. WANG ${ }^{1}$, M. CRANAGE ${ }^{2}$,

L.A. BERGMEIER ${ }^{1}$, E. MITCHELL ${ }^{1}$,

L. TAO ${ }^{1}$, G. HALL ${ }^{2}$, M. DENNIS ${ }^{2}$,

N. CoOK ${ }^{2}$, R. Brookes ${ }^{1}$, L. KLAVINSKIS ${ }^{1}$, I. JONES ${ }^{3}$, C. DOYLE ${ }^{3} \&$ R. WARD ${ }^{1}$

${ }^{1}$ Department of Immunology, United Medical and Dental School of Guy's and St. Thomas'

Hospital, Guy's Tower, Floor 28,

London SE1 9RT, UK

${ }^{2}$ Centre for Applied Microbiology and Research

Salisbury, Wiltshire SP4 OJG, UK

${ }^{3}$ Institute of Virology and Environmental

Microbiology, Oxford OX1 3SR, UK

1. Lehner, $T$. et al. Protective mucosal immunity elicited by targeted iliac lymph node immunization with a subunit SIV envelope and core vac- cine in macaques. Nature Med. 2, 767-776 (1996).

2. Müllbacher, A. \& Flynn, K. Aspects of cytotoxic T cell memory. Immunol. Rev. 150, 113-128 (1996).

3. Tough, D.F., Borrow, P. \& Sprent, J. Induction of bystander $\mathrm{T}$ cell proliferation by viruses and type 1 interferon in vivo. Science 272, 1947-1950 (1996).

4. Beverly, P.C.L. Human T cell memory. Curr. Top. Microbiol. Immunol. 159, 111 (1990).

5. Levy, J.A., Mackewicz, C.E. \& Barker, E. Controlling HIV pathogenesis: The role of the noncytotoxic anti-HIV response of CD8 ${ }^{*} \mathrm{~T}$ cells. Immunol. Today 17, 217 (1996).

6. Baier, M., Werner, A., Bannert, N., Metzner, K. \& Kurth, R. HIV suppression by interleukin-16. Nature 378, 563 (1995).

7. Bleul, C.C. et al. The lymphocyte chemoattractant SDF- 1 is a ligand for LESTR/fusin and blocks HIV-1 entry. Nature 382, 829 (1996).

8. Oberlin, E. et al. The CXC chemokine SDF-1 is the ligand for LESTR/fusin and prevents infection by T cell line adapted HIV-1. Nature 382, 833 (1996).

\title{
Cell status — dead or alive?
}

To the editor - In the July issue of Nature Medicine, Domenico Delia and colleagues reported that the sensitivity of human lymphoblastoid cells to $\gamma$-radiation was markedly affected by their p53 status, whereas this was not the case for their sensitivity to the chemotherapeutic agent Taxol $^{2}$. They examined EBV-immortalized lymphoblastoid cells from unrelated LiFraumeni syndrome patients heterozygous for p53 mutations (p53 wt/mut) and reported that resistance to radiation was markedly increased in p53 heterozygous cell lines relative to normal lymphoblastoid cells. They showed a plateau of survival as a function of dose between 800 and 1500 rads, with plateau survival values of $20 \%$ for the normal cells and $35 \%$ and $65 \%$ for the two heterozygous p $53 \mathrm{mu}$ tated cell lines. I suggest that the values quoted do not represent the true survival of the cells.

Ignoring for the moment the plateau in survival at doses above 800 rads (plateaus such as this have never been observed for radiation-induced cell killing), no human cell of any genotype, or tissue of origin, has been found to have a survival greater that $10 \%$ at doses of 1500 rads, and most have a survival of less than $0.1 \%$ at this dose. Since pioneering work showed that individual cells could be cloned in vitro and their survival following radiation assayed using the ability of the individual cells to form colonies", the so-called "clonogenic assay" has been used as the ultimate test of killing by radiation and chemotherapeutic drugs. Unfortunately clonogenic survival is not usually predicted by shortterm assays of cell viability based on cell numbers or by staining cells, because most mammalian cells do not die rapidly after radiation. In fact, they can go through up to four to five divisions after irradiation before eventually dying ${ }^{3}$. This is not only true for cells undergoing mitotically linked necrotic death, but also for cells undergoing apoptotic death following ionizing radiation ${ }^{4}$. Thus, Delia et al.'s assay for ionizing radiation (staining 24 hours after irradiation with Hoechst 33342 ) is unlikely to reflect the ultimate survival of the cells. Whether or not the data obtained for Taxol suffer from similar problems depends on the rate at which the cells die and leave the population after treatment with this agent.

Use of short-term assays, often based on the staining for apoptotic cells, is becoming increasingly prevalent in research on the sensitivity of cells to radiation and anticancer drugs. Unfortunately, interpretations based purely on these assays can lead to incorrect conclusions. For example, Aldridge et al. recently demonstrated that lymphoblastoid cells can differ markedly in their sensitivity to ionizing radiation, as judged by apoptosis, but show no change in the sensitivity of the cells, as judged by clonogenic sur$v^{\text {vival }}{ }^{5}$. Some of the growing confusion in the literature as to the effect of various genetic backgrounds on "sensitivity" to various agents might be resolved by a more careful examination of assays used.

\section{J. MARTIN BROWN \\ Division of Radiation Biology}

Department of Radiation Oncology

Stanford University

Stanford, California 94305-5468, USA 